\title{
Quantitative Analysis of Complex Drug-Drug Interactions between Cerivastatin and Metabolism/Transport Inhibitors Using Physiologically Based Pharmacokinetic Modeling ${ }^{\mathbb{}}$
}

\author{
Yoshiaki Yao, ${ }^{1}$ Kota Toshimoto, ${ }^{1}$ Soo-Jin Kim, Takashi Yoshikado, and Yuichi Sugiyama \\ Analysis \& Pharmacokinetics Research Laboratories, Drug Discovery Research, Astellas Pharma Inc., Ibaraki, Japan (Y.Y.), and \\ Sugiyama Laboratory, RIKEN Innovation Center, RIKEN, Kanagawa, Japan (K.T., S.K., T.Y., Y.S.)
}

Received October 29, 2017; accepted April 25, 2018

\begin{abstract}
Cerivastatin (CER) was withdrawn from the world market because of lethal rhabdomyolysis. Coadministrations of CER and cyclosporine A (CsA) or gemfibrozil (GEM) have been reported to increase the CER blood concentration. CsA is an inhibitor of organic anion transporting polypeptide (OATP)1B1 and CYP3A4, and GEM and its glucuronide (GEM-glu) inhibit OATP1B1 and CYP2C8. The purpose of this study was to describe the transporter-/enzyme-mediated drug-drug interactions (DDIs) of CER with CsA or GEM based on unified physiologically based pharmacokinetic (PBPK) models and to investigate whether the DDIs can be quantitatively analyzed by a bottom-up approach. Initially, the PBPK models for CER and GEM/GEM-glu were constructed based on the previously reported standard protocols. Next, the drug-dependent parameters were optimized by Cluster Newton Method. Thus, described
\end{abstract}

concentration-time profiles for CER and GEM/GEM-glu agreed well with the clinically observed data. The DDls were then simulated using the established PBPK models with previously obtained in vitro inhibition constants of CsA or GEM/GEM-glu against the OATP1B1 and cytochrome P450s. DDIs with the inhibitors were underestimated compared with observed data using the geometric means of reported values. To search for better described parameters within the range of in vitro values, sensitivity analyses were performed for DDIs of CER. Using the in vitro parameter sets selected by sensitivity analyses, these DDls were well reproduced, indicating that the present PBPK models were able to describe adequately the clinical DDIs based on a bottom-up approach. The approaches in this study would be applicable to the prediction of other DDIs involving both transporters and metabolic enzymes.
Introduction

Cerivastatin (CER), a potent 3-hydroxy-3-methylglutaryl coenzyme A (HMG-CoA) reductase inhibitor, was withdrawn from the world market because of the serious side effect of rhabdomyolysis (Wooltorton, 2001). In almost all lethal rhabdomyolysis cases, CER had been used with the lipid-lowering fibrate gemfibrozil (GEM) or

\footnotetext{
${ }^{1}$ Y.Y. and K.T. contributed to this research equally. https://doi.org/10.1124/dmd.117.079210.

SThis article has supplemental material available at dmd.aspetjournals.org.
}

prescribed at the highest therapeutic dose of $0.8 \mathrm{mg} / \mathrm{day}$ (Furberg and Pitt, 2001). Coadministration of GEM was reported to increase the CER blood concentrations (Backman et al., 2002). It has been reported that the risk of myopathy during treatment with statins depends on their dose or concentrations (Brewer, 2003). Given this, it has been widely speculated that the fatal rhabdomyolysis might be due in part to the increase in systemic exposure to CER, leading to its increase in muscle exposure.

CER is actively transported into the liver by organic anion transporting polypeptide (OATP)1Bs and then metabolized by two different P450 isoforms, CYP3A4 and CYP2C8 (Mück, 2000; Shitara et al., 2003;

ABBREVIATIONS: AUC, area under the blood concentration-time curve; $\beta$, ratio of hepatic and biliary excretion clearance to the sum of hepatic and biliary excretion clearance and passive diffusional clearance from the hepatocyte to the liver blood; CER, cerivastatin; $C_{\mathrm{h}}$, drug concentrations in the liver; $C L_{\text {int }}$, sum of intrinsic clearance of biliary excretion, hepatic metabolism, and glucuronidation; $C L_{\text {int,all, }}$, hepatic overall intrinsic clearance;

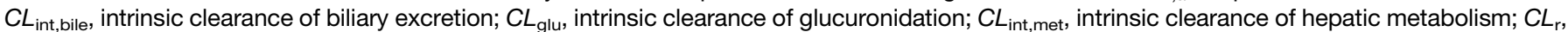
renal clearance; $C L_{\text {tot }}$, total clearance; CNM, Cluster Newton Method; CsA, cyclosporine A; $C_{\text {tissue, }}$ drug concentrations in tissue; DDIs, drug-drug interactions; DIN, a drug interaction number; $\mathrm{EH}$, hepatic extracellular space; EHC, enterohepatic circulation; $F_{\mathrm{a}} F_{\mathrm{g}}$, intestinal availability; $f_{\mathrm{b}}$, protein unbound fraction in blood; $\mathrm{f}_{\text {bile, }}$, contribution of biliary excretion to hepatic elimination; $f_{\text {glu }}$, contribution of glucuronidation to hepatic elimination; $F_{\mathrm{h}}$, hepatic availability; $f_{\mathrm{h}}$, hepatic protein unbound fraction; FLV, fluvastatin; $f_{\mathrm{m} \_\mathrm{CYP} 2 \mathrm{C} 8}$, fraction metabolized by CYP2C8 of CER; $f_{\mathrm{p}}$, protein unbound fraction in plasma; $\gamma$, ratio of influx intrinsic clearance by passive diffusion relative to efflux intrinsic clearance by passive diffusion through sinusoidal membrane; GEM, gemfibrozil; GEM-glu, gemfibrozil 1-O- $\beta$-glucuronide; HC, hepatocytes; HMG-CoA, 3-hydroxy-3-methylglutaryl coenzyme $\mathrm{A} ; k_{\mathrm{a}}$, absorption rate constant; $k_{\text {bile, }}$, biliary excretion rate constant; $\mathrm{k}_{\text {deconj }}$, deconjugation rate constant of gemfibrozil 1 - $\mathrm{O}$ $\beta$-glucuronide; $\mathrm{k}_{\text {deg_CYP2C8, }}$, degradation rate constant of CYP2C8; $k_{\mathrm{f}}$, fecal elimination rate constant; $K_{\mathrm{i}}$, inhibition constant; $\mathrm{K}_{\mathrm{i}, \mathrm{app}} \mathrm{CYP2C8}$, apparent dissociation constant between CYP2C8 and the inhibitor; $k_{\text {inact }}$, maximal inactivation rate constant; $K_{\mathrm{p}}$, tissue to blood concentration ratio; $k_{\text {transit }}$, transit rate constant for intestinal compartment; OATP, organic anion transporting polypeptide; P450, cytochrome P450; PBPK, physiologically

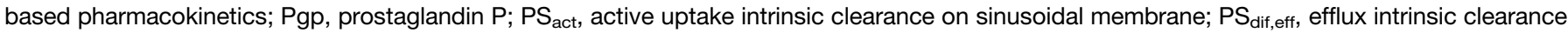

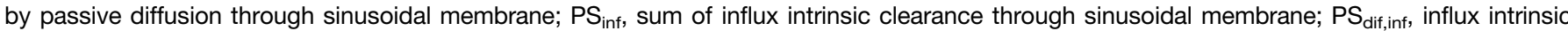

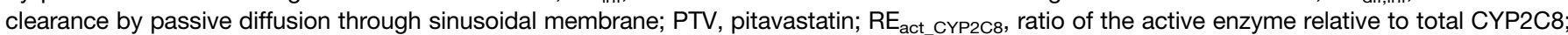
WSS, weighted sum of squares. 
Shitara and Sugiyama, 2006). It was previously reported that CER concentrations were increased 2.5-to 5.0-fold by the concomitant use of GEM, as mentioned above, and by the treatment with the immunosuppressive agent cyclosporine A (CsA) in kidney transplant patients (Mück et al., 1999; Backman et al., 2002). CsA markedly inhibits OATP1B1-mediated hepatic uptake and, to a smaller extent, CYP3A4. GEM and its metabolite, GEM-glucuronide (GEM-glu), also moderately inhibit OATP1B1. Furthermore, GEM-glu irreversibly inhibits CYP2C8-mediated metabolism (Shitara et al., 2003; Ogilvie et al., 2006; Varma et al., 2015). Thus, the pharmacokinetic alteration under DDIs of CER is suggested to be caused by both transporter- and P450-mediated inhibition. Figure 1A shows the qualitative DDI mechanisms of CER with CsA or GEM in the liver.

Quantitative analyses of such complex DDIs, as in the case for CER, are challenging but essential for adequate assessment of the clinical use of drugs, particularly regarding safety and effectiveness. Regulatory agencies strongly recommend the evaluation of DDI potential with a mechanistic rationale for sponsors during drug development. In the regulatory guidelines/guidance on DDIs, the usefulness of the PBPK modeling approach is mentioned as a powerful tool to guide rationalized design of DDI studies and to quantitatively predict the potential of DDIs (https://www.fda.gov/downloads/drugs/guidancecomplianceregulatoryinformation/guidances/ucm292362.pdf; http://www.ema.europa.eu/docs/ en_GB/document_library/Scientific_guideline/2012/07/WC500129606. pdf; https://www.pmda.go.jp/files/000206158.pdf). On the other hand, the regulatory authorities also document that the robustness of PBPK models should be evaluated by performing verification and modification since the choice of structural models and availability of drugdependent parameters are key issues in these analyses (https://www. fda.gov/downloads/drugs/guidancecomplianceregulatoryinformation/ guidances/ucm531207.pdf; http://www.ema.europa.eu/docs/en_GB/ document_library/Scientific_guideline/2016/07/WC500211315.pdf).

Recently, our group proposed a standard scheme of PBPK analyses to predict quantitatively the DDIs primarily caused by the OATPsmediated hepatic uptake (Yoshikado et al., 2016). In that study, similar inhibition constants $\left(K_{\mathrm{i}}\right)$ against OATP1Bs of inhibitors CsA or rifampicin were obtained regardless of the substrates, pitavastatin (PTV), or fluvastatin (FLV). In addition, the obtained in vivo $K_{\mathrm{i}}$ values of CsA against OATP1Bs were comparable with in vitro $K_{\mathrm{i}}\left(\right.$ or $\left.\mathrm{IC}_{50}\right)$ values in the presence of CsA preincubation. These results suggested the validity of the PBPK models of these drugs and the effectiveness of the drug-dependent parameter optimization scheme for predicting other DDIs involving these drugs.

The purpose of this study was to construct unified PBPK models to analyze transporter- and enzyme-mediated complex DDIs of CER with CsA or GEM based on the standard protocol. Because there are some unknown parameters whose initial values were difficult to set directly in the models because of limited information, the PK parameters of CER or GEM were estimated by a parameter estimation algorithm called the Cluster Newton Method (CNM) (Aoki et al., 2014). The CNM has the following advantages: it requires only the designation of relatively broad parameter ranges as an initial setting, whereas the conventional algorithm, such as the Levenberg-Marquardt method, requires identification of feasible initial parameters, and the results are obtained as multiple sets of optimized parameters. Previous studies have reported that CNM has been successfully applied in analyzing complex DDIs using PBPK models (Yoshida et al., 2013; Toshimoto et al., 2017).

Moreover, to investigate whether DDIs of CER can be quantitatively described by unified PBPK models based on a bottom-up approach, simulations for DDIs with CsA and GEM were carried out using in vitro values. It was reported that the in vitro inhibition-related values of the drugs for OATP1B1 or cytochromes P450s varied widely, possibly affected by experimental conditions (Izumi et al., 2013, 2015). To evaluate the validity of these in vitro values and to select the well described parameter sets, therefore, sensitivity analyses for the values were carried out for DDIs of CER.

\section{Materials and Methods}

\section{Construction of PBPK Models}

The PBPK models were constructed to describe the blood concentration-time profiles of CER or GEM/GEM-glu according to their PK properties based on previously reported standard models with some modifications (Yoshikado et al., 2016). The model structures are shown in Fig. 1, B and D. The liver was divided into five compartments for hepatic extracellular and hepatocellular parts to reproduce the hepatic availability $\left(F_{\mathrm{h}}\right)$ of dispersion model. Rapid equilibrium in the muscle, skin, and adipose, whose distribution volume is not negligible, was also included. In addition, to represent the delay of the absorption process, an empirical transit compartment was added in connection with the intestinal compartment.

In the CER and GEM-glu case, to represent the enterohepatic circulation (EHC), three transit compartments between the compartments for hepatocytes and the intestine were incorporated. In the GEM/GEM-glu case, as a rapid equilibrium distribution of GEM in the liver could be assumed from the previous report, compartments for hepatic extracellular spaces and hepatocytes were unified into a single liver compartment (Kimoto et al., 2015). Since GEM-glu was reported to be a substrate for OATPs (1B1, 1B3, and 2B1), the GEM-glu was also assumed to be absorbed from the intestine (Kimoto et al., 2015). Moreover, glucuronidation and deconjugation were assumed to occur only in the liver and the intestine, respectively. The CsA PBPK model (Fig. 1C) and reported PK parameters were used in this study (Yoshikado et al., 2016).

\section{Calculation of PK Parameters of CER, GEM, and GEM-glu}

Based on the standard protocol that was previously proposed (Yoshikado et al., 2016), PK parameters of CER and GEM/GEM-glu were collected from the literature, in silico calculations, our experiments and calculation methods as shown in Supplemental Tables 1 and 3 (Davies and Morris, 1993; Mück et al., 1997; Shitara et al., 2003, 2004; Shitara and Sugiyama, 2006; Yang et al., 2008; Watanabe et al., 2009, 2011; Deguchi et al., 2011; Honkalammi et al., 2011; Kudo et al., 2013; Yoshikado et al., 2017). Some hybrid parameters ( $C L_{\text {int,all }}, \beta, \gamma$, and $f_{\text {bile }}$ ) were used instead of intrinsic clearances $\left(P S_{\text {act }}, P S_{\text {dif,inf }}, P S_{\text {dif,eff, }}, C L_{\text {int,bile }}\right.$, and $C L_{\text {int,met }}$ ) in the same way as previously reported (Supplemental Tables 1 and 3; Tables 1 and 2) (Yoshikado et al., 2016). $K_{\text {p,liver }}$ of GEM was calculated based on the reported in silico method (Rodgers and Rowland, 2006) that uses $\operatorname{cog} \mathrm{P}$ and $\mathrm{pK}_{\mathrm{a}}$ described in Scifinder Scholar (Chemical Abstracts Service, Columbus, $\mathrm{OH})$. Hybrid parameters were defined as follows in eq. 1-5:

$$
\begin{aligned}
& f_{b} \times C L_{\text {int }, \text { all }}=f_{b} \times\left(P S_{a c t}+P S_{\text {dif }, \text { inf }}\right) \\
& \times \frac{f_{h} \times C L_{\text {int }, \text { met }}+f_{h} \times C L_{\text {int }, \text { bile }}}{f_{h} \times P S_{\text {dif }, \text { eff }}+f_{h} \times C L_{\text {int }, \text { met }}+f_{h} \times C L_{\text {int }, \text { bile }}} \\
& R_{\text {dif }}=\frac{P S_{\text {dif }, i n f}}{P S_{\text {act }}} \\
& \beta=\frac{f_{h} \times C L_{\text {int,met }}+f_{h} \times C L_{\text {int }, \text { bile }}}{f_{h} \times P S_{\text {dif, }, \text { ff }}+f_{h} \times C L_{\text {int, met }}+f_{h} \times C L_{\text {int }, \text { bile }}} \\
& \gamma=\frac{P S_{\text {dif, inf }}}{P S_{\text {dif }, \text { eff }}} \\
& f_{\text {bile }}=\frac{C L_{\text {int }, \text { bile }}}{C L_{\text {int }, \text { met }}+C L_{\text {int }, \text { bile }}}
\end{aligned}
$$

Initial ranges of unknown PK parameters for CNM optimization are shown in Tables 1 and 2. All drug-dependent parameters were calculated according to the methods reported by our group (Yoshikado et al., 2016; Kim et al., 2017; Toshimoto et al., 2017).

Parameter Optimization by the CNM. The PK parameters of CER and GEM/GEM-glu in the PBPK models were optimized by fitting to their blood concentration profiles using CNM (Mück et al., 1997; Honkalammi et al., 2011; Toshimoto et al., 2017). The optimization was carried out as described in the report by Toshimoto et al. (2017) with some modifications. Briefly, 1000 initial 
A

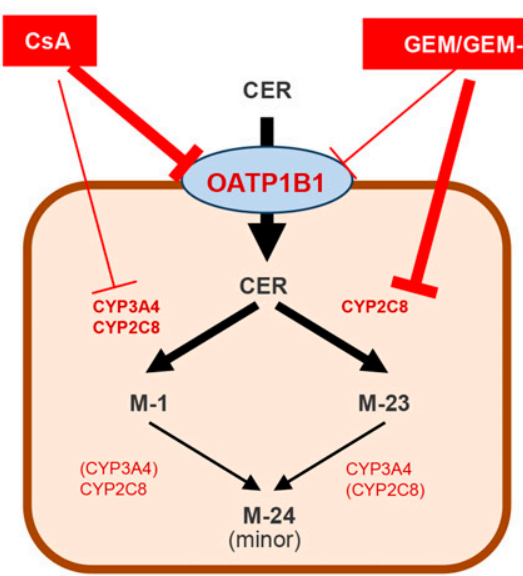

B

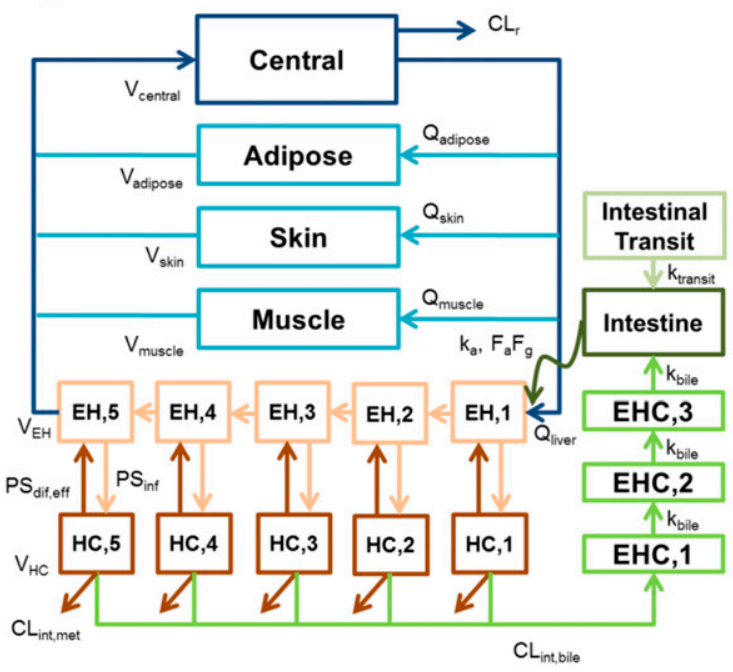

C

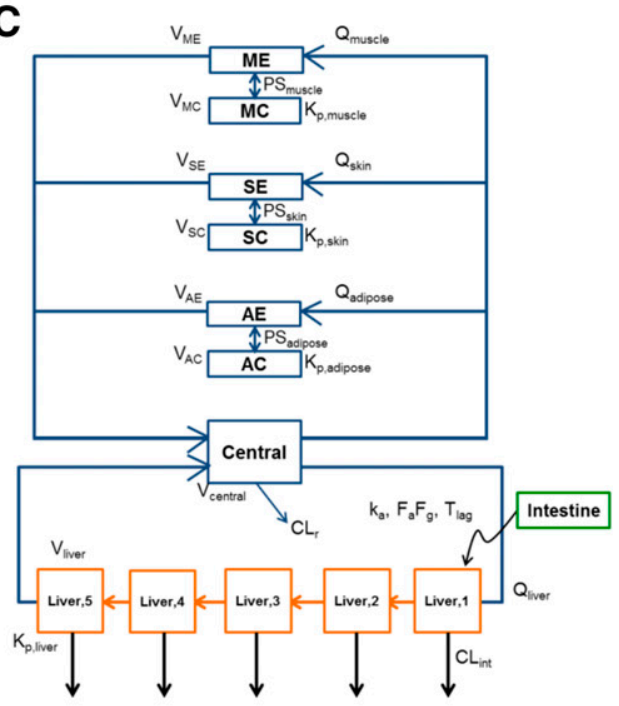

D

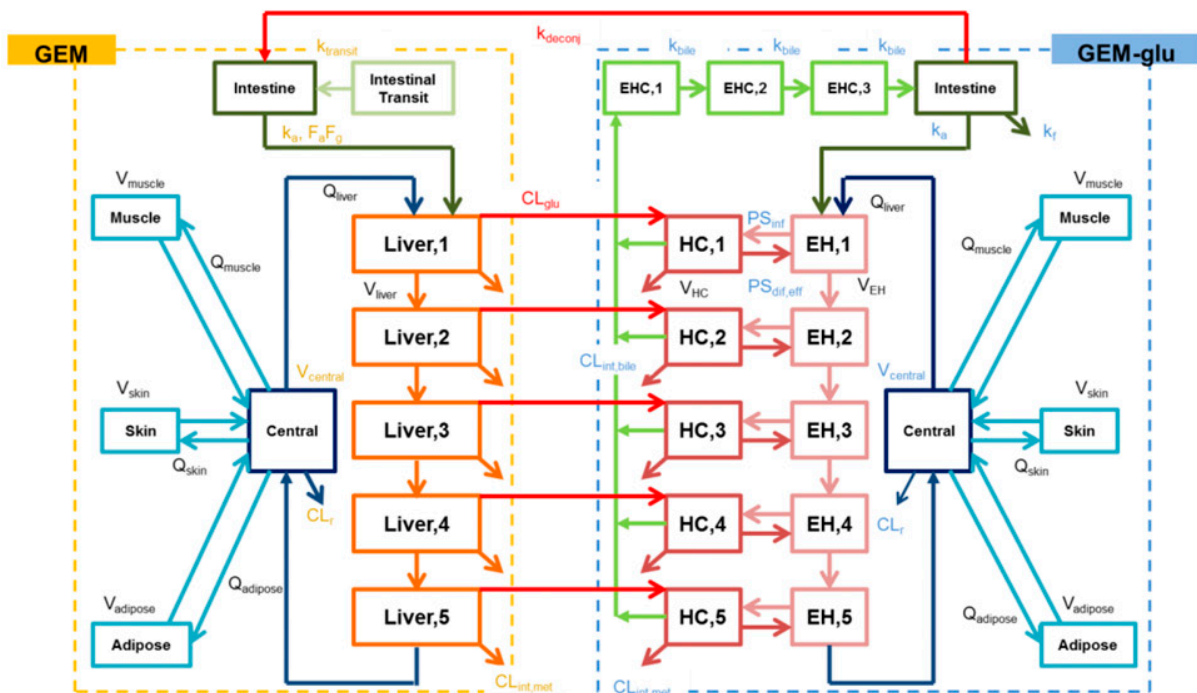

Fig. 1. Diagram of DDI in the liver (A) and the structures of PBPK models (B-D). (A) CER is transported into the liver by OATP1B1 and then metabolized by CYP2C8 and CYP3A4. Coadministration of CER and CsA, an inhibitor of OATP1B1 and CYP3A4 or GEM, inhibitors of OATP1B1 and CYP2C8 as GEM and its glucuronide (GEM-glu)) increases the AUC and $C_{\max }$ of CER. (B-D) Structures of the PBPK models for CER (B), CsA (C), and GEM/GEM-glu (D). These models were based on previously reported models with some modifications (Yoshikado et al., 2016).

sets of parameters, whose ranges are described in Table 1 (CER) and Table 2 (GEM/GEM-glu), were first generated and optimized. The area under the blood concentration-time curve (AUC) value of CER after intravenous administration or the values of GEM and GEM-glu after oral administration of GEM were used as the objective function for optimization. Procedures for parameter generation and optimization were repeated 50 times with 1000 different initial sets. Next, the 
TABLE 1

Average, S.D., and coefficient of variant (CV\%) of the 30 most optimized drugdependent parameter sets of CER selected to minimize the WSS from the observed i.v. and p.o. data after the optimization of model parameters by CNM to explain the pharmacokinetic data under i.v. conditions

\begin{tabular}{lccccr}
\hline Parameters & Units & Initial Range & Average & S.D. & CV\% \\
\hline$V_{\text {central }}$ & $\mathrm{L} / \mathrm{kg}$ & $0.075-0.75$ & 0.092 & 0.039 & 42.5 \\
$k_{\mathrm{a}}$ & $\mathrm{h}$ & $0.06-6$ & 3.249 & 1.274 & 39.2 \\
$k_{\text {transit }}$ & $/ \mathrm{h}$ & $0.06-6$ & 0.769 & 0.535 & 69.6 \\
$1 / \beta$ & & $1.1-10$ & 2.358 & 1.643 & 69.7 \\
$f_{\mathrm{b}} * \mathrm{CL}_{\text {int,all }}$ & $\mathrm{L} / \mathrm{h} / \mathrm{kg}$ & $0.0372-3.72$ & 0.638 & 0.033 & 5.2 \\
$k_{\text {bile }}$ & $/ \mathrm{h}$ & $0.06-6$ & 4.029 & 1.082 & 26.9 \\
$1 / f_{\text {bile }}$ & & $1.1-10$ & 1.411 & 0.087 & 6.2 \\
\hline
\end{tabular}

blood concentration-time profiles of CER and GEM/GEM-glu were simulated using the 50,000 optimized parameter sets. The weighted sum of squares (WSS) between the simulated and the observed profile was calculated as described in the following (eq. 6):

$$
W S S=\sum_{i=1}^{n} \frac{\left(y_{i}-y_{i}^{\prime}\right)^{2}}{y_{i}{ }^{2}} y_{i}: i \text { th observed value, } y_{i}^{\prime}: \text { ith predicted value }
$$

In the case of CER, the top 30 drug-dependent parameter sets were selected to minimize the WSS between the simulated and observed profiles after oral and intravenous administration, and the average of 30 selected parameter sets was used for subsequent DDI analyses. In the case of GEM/GEM-glu, some parameters were correlated to each other; thus, the top five drug-dependent parameter sets were selected to minimize the WSS between the simulated and observed profiles of GEM and GEM-glu after oral administration of GEM, and each parameter set was used for subsequent DDI analysis.

\section{Parameter Optimization by Nonlinear Least-Squares Fitting}

PK parameters of CER under control conditions and those of CsA in the DDI studies in the PBPK models were reoptimized by fitting to their blood concentration profiles (Mück et al., 1999; Backman et al., 2002) using the nonlinear least squares fitting software Napp (version 2.31) (Hisaka and Sugiyama, 1998). The weight for the calculation was set as 1 (the square root of the value).

\section{Simulation of CER Concentration Profiles under DDI Conditions}

In this study the competitive inhibition of CsA against OATP1B1 and CYP3A4 and that of GEM/GEM-glu against OATP1B1, as well as mechanism-based inhibition of GEM-glu against CYP2C8, were incorporated into the analyses. CER is metabolized by CYP3A4 and CYP2C8 (Mück, 2000; Shitara et al., 2003; Shitara and Sugiyama, 2006), so the sum of $\mathrm{f}_{\mathrm{m}_{-} \mathrm{CYP} 2 \mathrm{C} 8}$ and $\mathrm{f}_{\mathrm{m}_{-} \mathrm{CYP} 3 \mathrm{~A} 4}$ was assumed to be 1 . The blood concentration-time profile of CER under DDI conditions with CsA was simulated with consideration to the inhibition of a hepatic uptake and competitive inhibition of CYP3A4 expressed by eq. 7 and 8:

Inhibition of OATP1B1-Mediated Hepatic Uptake.

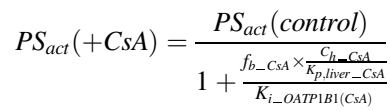

Inhibition of P450-Mediated Metabolism.

$$
\begin{aligned}
C L_{\text {int }, \text { met }}(+C s A)= & C L_{\text {int }, \text { met }}(\text { control }) \\
& \times\left(f_{m_{-} C Y P 2 C 8}+\frac{1-f_{m_{-} C Y P 2 C 8}}{1+\frac{f_{h_{-} C s A} \times C_{h_{-} C s A}}{K_{i_{-} C Y P 3 A 4}(C S A)}}\right)
\end{aligned}
$$

where $P S_{a c t}$ represents the intrinsic clearance for hepatic active uptake of CER from blood; $K_{p, \text { liver_CsA }}$ represents liver-to-blood concentration ratio of CsA; $C L_{\text {int,met }}$ represents the intrinsic clearance for hepatic metabolism mediated by CYP2C8 and CYP3A4 of CER; $f_{b_{-} C s A}$ and $f_{h_{-} C s A}$ represent the unbound fraction of $C s A$ in the blood and the liver compartment, respectively; $C_{h_{-} C s A}$ represents the concentration of $C s A$ in the liver compartment; $f_{m_{-} C Y P 2 C 8}$ represents the fraction metabolized by $C Y P 2 C 8$ of $C E R$; and $K_{i \_O A T P 1 B 1(C S A)}$ and $K_{i \_C Y P 3 A 4(C s A)}$ represent the inhibition constant of $C s A$ for OATP1B1-mediated hepatic uptake and CYP3A4-mediated hepatic metabolism, respectively.

Similarly, the blood concentration-time profile of CER under DDI conditions with GEM was simulated considering the inhibition of hepatic uptake by GEM and GEM-glu and a mechanism-based inhibition of CYP2C8 by GEM-glu expressed by eq. 9 and 10 :

Inhibition of OATP1B1-Mediated Hepatic Uptake.

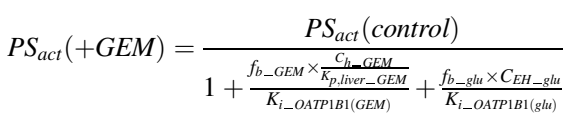

Inhibition of P450-Mediated Metabolism.

$$
\begin{aligned}
C L_{\text {int }, \text { met }}(+G E M)= & C L_{\text {int }, \text { met }}(\text { control }) \times\left(f_{m-C Y P 2 C 8} \times R E_{\text {act }-C Y P 2 C 8}\right. \\
& \left.+\left(1-f_{m-C Y P 2 C 8}\right)\right)
\end{aligned}
$$

where $f_{b_{-} G E M}$ and $f_{b_{\_} g l u}$ represent the unbound fraction in the blood of GEM and GEM-glu, respectively; $K_{p, \text { liver_GEM }}$ represents liver-to-blood concentration ratio of GEM; $C_{h_{-} G E M}$ represents the concentration of GEM in the liver compartment; $C_{E H_{-} g l u}$ represents the concentration of GEM-glu in the hepatic extracellular space compartment; $R E_{\text {act_CYP2C8 }}$ represents the ratio of the active enzyme relative to

\begin{tabular}{|c|c|c|c|c|c|c|c|c|}
\hline & Parameters & Units & Initial Range & $1 \mathrm{st}$ & 2nd & $3 \mathrm{rd}$ & 4th & 5th \\
\hline \multirow[t]{6}{*}{ GEM } & $V_{\text {central }}$ & $\mathrm{L} / \mathrm{kg}$ & $0.075-0.75$ & 0.077 & 0.075 & 0.077 & 0.089 & 0.076 \\
\hline & $k_{\mathrm{a}}$ & $/ \mathrm{h}$ & $0.06-6$ & 1.463 & 2.574 & 1.611 & 1.275 & 4.733 \\
\hline & $k_{\text {transit }}^{a}$ & $/ \mathrm{h}$ & $0.06-6$ & 3.645 & 2.872 & 2.378 & 6.585 & 1.488 \\
\hline & $f_{\mathrm{b}} * \mathrm{CL}_{\text {int,all }}$ & $\mathrm{L} / \mathrm{hr} / \mathrm{kg}$ & $0.0105-1.05$ & 0.157 & 0.173 & 0.165 & 0.154 & 0.199 \\
\hline & $1 / f_{\mathrm{glu}}$ & & $1.1-10$ & 1.210 & 1.118 & 1.263 & 1.105 & 1.273 \\
\hline & $V_{\text {central }}$ & $\mathrm{L} / \mathrm{kg}$ & $0.075-0.75$ & 0.076 & 0.076 & 0.087 & 0.150 & 0.088 \\
\hline \multirow{7}{*}{ GEM-glu } & & $/ \mathrm{h}$ & $0.06-6$ & 0.258 & 0.109 & 0.106 & 0.155 & 0.142 \\
\hline & $R_{\mathrm{dif}}$ & & $0.0275-0.11$ & 0.051 & 0.103 & 0.064 & 0.079 & 0.096 \\
\hline & $1 / \beta$ & & $1.1-10$ & 5.727 & 2.119 & 6.318 & 14.039 & 2.779 \\
\hline & $f_{\mathrm{b}} * C L_{\text {int,all }}$ & $\mathrm{L} / \mathrm{hr} / \mathrm{kg}$ & $0.0156-1.56$ & 0.245 & 0.159 & 0.293 & 0.314 & 0.184 \\
\hline & $k_{\text {bile }}$ & $/ \mathrm{h}$ & $0.06-6$ & 0.205 & 0.209 & 2.235 & 0.095 & 3.807 \\
\hline & $k_{\text {deconj }}$ & $/ \mathrm{h}$ & $0.06-6$ & 0.856 & 1.179 & 0.073 & 0.189 & 0.824 \\
\hline & $k_{\mathrm{f}}$ & $/ \mathrm{h}$ & $0.06-6$ & 0.938 & 1.414 & 0.906 & 0.067 & 2.466 \\
\hline WSS & & & & 1.202 & 1.319 & 1.514 & 1.521 & 1.586 \\
\hline
\end{tabular}
total CYP2C8; and $K_{i_{-} O A T P 1 B 1(G E M)}$; and $K_{i_{-} O A T P 1 B 1(g l u)}$ represents the inhibition constant for OATP1B1-mediated hepatic uptake of GEM and GEM-glu, respectively.

TABLE 2

Optimized top five drug-dependent parameter sets of GEM/GEM-glu selected to minimize the weighted sum of squares (WSS) from the observed clinical data by Cluster Newton Method (CNM) 
i.v.

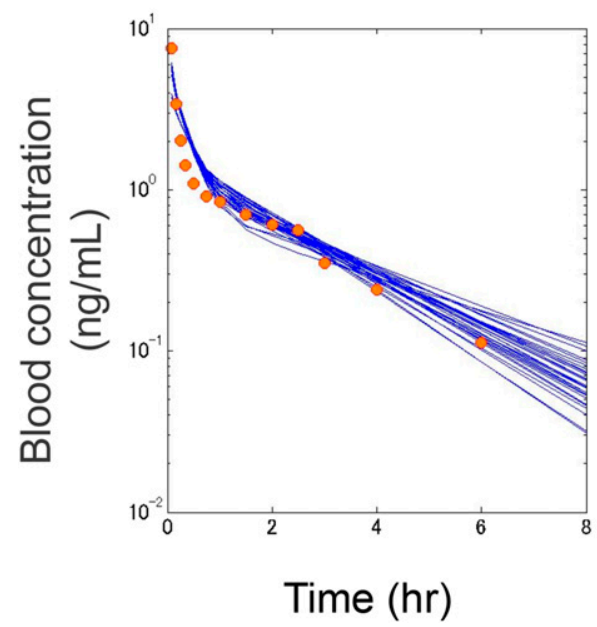

p.o.

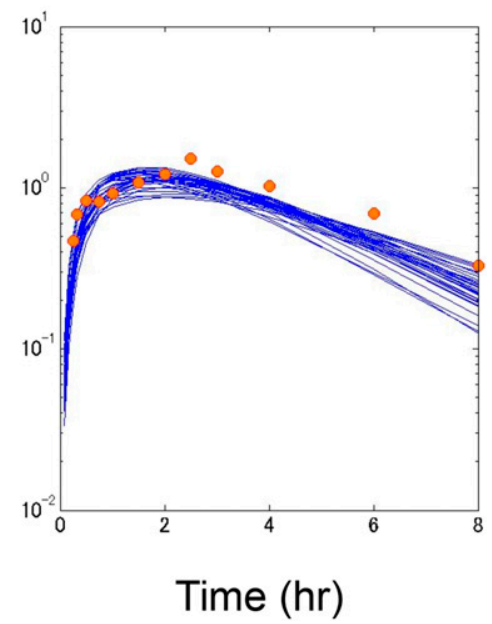

Fig. 2. Blood concentration-time profile of CER after single i.v. $(0.1 \mathrm{mg})$ or p.o. $(0.2 \mathrm{mg})$ administration. Fitting analysis was performed by CNM. Solid lines represent the fitted time course results using the 30 most optimized drug-dependent parameter sets selected to minimize the WSS from the observed intravenous and oral data after optimization of model parameters to explain the PK data under intravenous conditions. The closed circles represent the observed values (Mück et al., 1997). Optimized parameters are described in Table 1. Fixed pharmacokinetic parameters are described in Supplemental Table 1.
Differential equation (eq. 11) for $\mathrm{RE}_{\text {act_CYP2C8 }}$ is expressed as follows considering that the mechanism-based inhibition by GEM-glu

$$
\begin{aligned}
& \frac{d R E_{a c t \_C Y P 2 C 8}}{d t}=-\frac{k_{\text {inact_C } C P P 2 C 8(g l u)} \times R E_{a c t-C Y P 2 C 8} \times f_{h_{-} g l u} \times C_{H C_{-} g l u}}{K_{i, a p p_{-} C Y P 2 C 8(g l u)}+f_{h_{-} g l u} \times C_{H C_{-} g l u}} \\
& +k_{\text {deg_C }_{-} Y P 2 C 8} \times\left(1-R E_{\text {act_CYP2C8 }}\right) \text {, }
\end{aligned}
$$

where $k_{\text {inact } C Y P 2 C 8(g l u)}$ represents the maximum inactivation rate constant by GEM-glu; $K_{i, a p p \_C Y P 2 C 8(g l u)}$ represents the apparent dissociation constant between CYP2C8 and GEM-glu; $C_{H_{C} \text { glu }}$ represents the concentration of GEM-glu in the hepatocytes compartment; $f_{h \_g l u}$ represents the unbound fraction of GEM-glu in the hepatocytes compartment; and $k_{\text {deg_CYP2C8 }}$ represents the degradation rate constant of CYP2C8.

All in vitro inhibition constant $\left(K_{\mathrm{i}}, K_{\mathrm{i}, \text { app }}\right.$, or $\left.\mathrm{IC}_{50}\right)$ values were derived from the University of Washington's Metabolism and Transport Drug Interaction Database (http://www.druginteractioninfo.org) for each inhibitor against the specific enzyme/transporter in various experimental conditions (Supplemental Table 5) (Kim et al., 2017). The differential equations used in these analyses are shown in Supplemental Text.

\section{Results}

Analysis of the Blood Concentration-Time Profile of CER. To obtain the optimized drug-dependent parameters of CER in the PBPK model (Fig. 1B), its blood concentration-time profile was first fitted by CNM to the clinically observed data after single intravenous administration of CER (Mück et al., 1997). Fixed parameters used in the analysis are summarized in Supplemental Table 1. The 30 most optimized parameter sets were then selected to minimize the WSS from the observed PK data of single intravenous and oral administration of CER (Mück et al., 1997). The averages of the 30 most-optimized parameter sets are described in Table 1. As shown in Fig. 2, the reproduced concentration profiles after single intravenous and oral administration of CER agreed well with the clinically observed data (Mück et al., 1997).

Analysis of the Blood Concentration-Time Profile of CsA. To perform the DDI analysis of CER with CsA, the PK parameters, except $k_{\mathrm{a}}, f_{\mathrm{h}} * C L_{\mathrm{int}}$ and dose (there is no information about dose in the literature), of CsA were reoptimized based on the previously reported PBPK model (Fig. 1C) (Yoshikado et al., 2016) with the clinically observed data of the kidney transplant patients receiving stable CsA treatment (Mück et al., 1999) using Napp. All parameters, except $k_{\mathrm{a}}, f_{\mathrm{h}}{ }^{*} \mathrm{CL}_{\mathrm{int}}$, and dose, were the same as previously reported (Yoshikado et al., 2016). In that study,
CsA was stably orally administered with steady state defined as unaltered CsA dosing and blood concentration of $90-120 \mathrm{ng} / \mathrm{ml}$ according to the local regimen at stable transplant function for at least 1 month, assessed by at least three blood-level measurements. Thus, the fitting analysis was performed with twice-daily administration of CsA for 6 days (under steady-state conditions). Optimized parameters and fitted concentration-time profiles are shown in Supplemental Table 2 and Supplemental Fig. 1, respectively. Estimated $f_{\mathrm{h}} * C L_{\text {int }}$ was similar to initial values, within 1.5 times (Supplemental Table 2), and the reproduced concentration profile of CsA agreed well with the clinically observed data (Mück et al., 1999).

Fitting Analysis of the Blood Concentration-Time Profile of GEM/GEM-glu. To perform the DDI analysis of CER with GEM, the blood concentration-time profiles of GEM and GEM-glu were fitted by CNM based on the PBPK model (Fig. 1D) to the clinically observed PK data after single oral administration of GEM (Honkalammi et al., 2011). Fixed PK parameters used in the analysis are summarized in Supplemental Table 3. Since there are some correlated parameters and the average value cannot be used in this case, the five most-optimized drugdependent parameter sets were selected to minimize the WSS from the observed PK data of GEM and GEM-glu (Honkalammi et al., 2011) to perform DDI analyses using each five-parameter set. Optimized parameter sets are described in Table 2. As shown in Fig. 3, the reproduced concentration profiles of GEM and GEM-glu after oral administration of GEM agreed well with the clinically observed data (Honkalammi et al., 2011). Only the best described result of GEM/GEM-glu was used in initial DDI simulation and sensitivity analyses, although final DDI simulation using the five best described drug-dependent parameter sets after sensitivity analyses were performed in each five case.

Simulation of the Blood Concentration-Time Profile of CER under DDI Conditions with CsA or GEM. To perform the DDI analysis of CER with CsA or GEM, the blood concentration-time profiles of CER under control conditions were first reproduced by reoptimization using Napp software of the values of $k_{\mathrm{a}}, f_{\mathrm{b}} * C L_{\text {int,all }}$, and $k_{\text {bile }}$ based on the PBPK model with the average of the obtained 30 most optimized drug-dependent parameter sets as initial values (Fig. 1B). Optimized parameters and fitted concentration-time profiles of CER are shown in Supplemental Table 4 and Supplemental Fig. 2, respectively. Estimated values of $f_{\mathrm{b}} * C L_{\text {int, all }}$ in both cases were similar to 
GEM
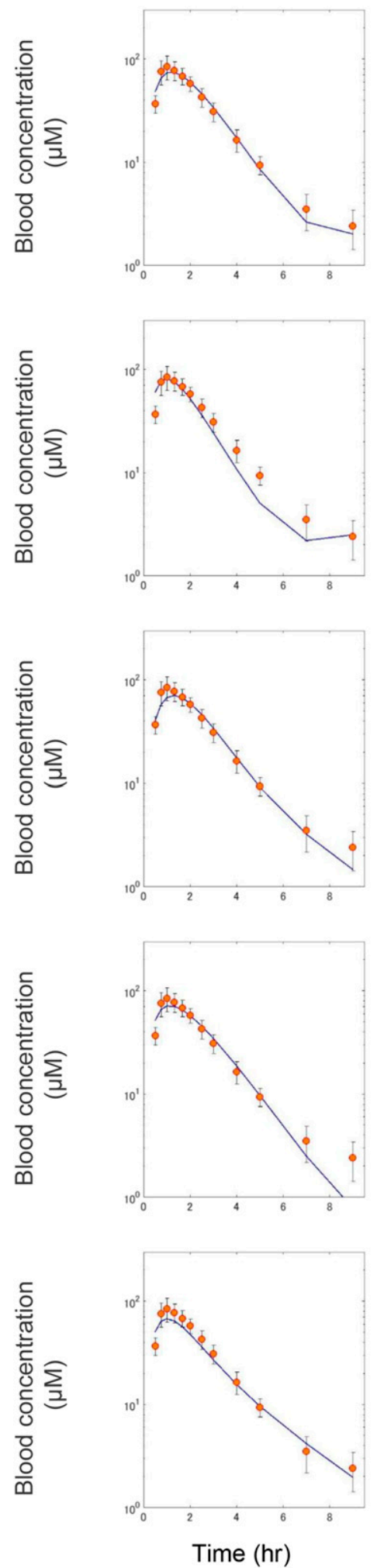

GEM-glu
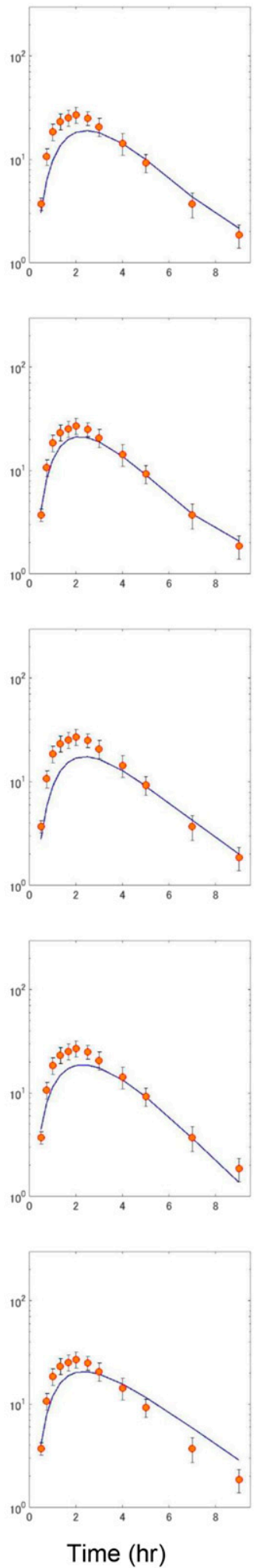

1 st

2nd

Fig. 3. Blood concentration-time profile of GEM/GEM-glu after single oral administration of GEM (600 mg). Fitting analysis was performed by the CNM. Solid lines represent the fitted time course results using the first to fifth most optimized drug-dependent parameter sets, which were selected to minimize the WSS from the observed PK data of GEM/GEM-glu. Closed circles represent the observed values (Honkalammi et al., 2011). Optimized parameters are described in Table 2. Fixed PK parameters are described in Supplemental Table 3.

initial values, within 1.2 times (Supplemental Table 4), and the obtained concentration profiles of CER under control conditions agreed well with the clinically observed data (Mück et al., 1999; Backman et al., 2002). Subsequently, the CER concentration profiles under DDI conditions with CsA or GEM were simulated, as shown in Supplemental Fig. 3, based on the PBPK models (Figs. 1B-D) using the geometric mean of in vitro inhibition constants (Supplemental Table 5) (Kim et al., 2017), together with the drug-dependent parameters estimated in the present study described in Tables 1 and 2, and Supplemental Tables 2 and 4. In the case of DDI with CsA, the in vivo $K_{\mathrm{i}}$ value of CsA against 
OATP1B1-mediated transport of PTV or FLV was reported to be comparable with in vitro $K_{\mathrm{i}}$ (or $\mathrm{IC}_{50}$ ) values in the presence of CsA preincubation (Li et al., 2014; Yoshikado et al., 2016). In this study, therefore, in vitro $K_{\mathrm{i}}$ (or $\mathrm{IC}_{50}$ ) values of CsA with preincubation against OATP1B1 were used for DDI analysis. Furthermore, in the case of DDI with GEM the ratio of $K_{\mathrm{i}_{-} \mathrm{OATP} 1 \mathrm{~B} 1 \text { (glu) }}$ to $K_{\mathrm{i}_{-} \mathrm{OATP} 1 \mathrm{~B} 1 \text { (GEM) }}$ was fixed at the ratio of the geometric means of the reported in vitro value (0.371) (Kim et al., 2017) to simplify the analysis, using the same technique as Kudo et al. (2013). As shown in Supplemental Fig. 3A, simulated $C_{\max }$ of the CER concentration profile was lower than the observed data in DDI with CsA. Similarly, as shown in Supplemental Fig. 3B, the $C_{\max }$ and $t_{1 / 2}$ were underestimated in DDI with GEM.

Sensitivity Analyses of the Parameters of CER and CsA Under DDI Conditions with CsA. To search for better described DDI-related parameters of CER with CsA from reported in vitro values, sensitivity analyses were carried out for $K_{\mathrm{i}_{\_} \text {OATP1B } 1}$ and $K_{\mathrm{i}_{-} \text {CYP3A4 }}$ of CsA under DDI condition with CsA (Supplemental Figs. 4A and B). Upper and lower limits of the range of inhibition constants were set by maximum and minimum value of the respective in vitro constant (Supplemental Table 5) (Kim et al., 2017). As shown in Supplemental Figs. 4A and B, the simulated CER concentration profile was changed by alteration of $K_{\mathrm{i} \_ \text {OATP1B1 }}$ but was hardly changed by $K_{\mathrm{i} \_ \text {CYP3A4 }}$. To examine a relationship between the blood/liver concentration of CsA and the in vitro $K_{\mathrm{i}}$ value, unbound blood and intrahepatic concentration-time profiles of CsA were simulated (Supplemental Fig. 4E) using CsA parameters that have been previously reported and in Supplemental Table 2. The simulated unbound CsA blood concentration was comparable to the in vitro $K_{\mathrm{i} \_ \text {OATP1B1 }}$, whereas the intrahepatic unbound concentration was much lower than the in vitro $K_{\mathrm{i} \_ \text {CYP3A4 }}$.

It is reported that CER is a substrate of P-glycoprotein (Pgp) (Kivistö et al., 2004) and CsA is an inhibitor of Pgp (Yoshida et al., 2012), indicating that CsA might also inhibit intestinal Pgp and that this inhibition might increase the magnitude of DDI. Yet, in these DDI analyses, intestinal inhibition of Pgp or CYP3A4 are not incorporated. Accordingly, sensitivity analyses were performed to take account of intestinal Pgp and CYP3A4 inhibition by changing $F_{\mathrm{a}} F_{\mathrm{g}}$ and/or $k_{\mathrm{a}}$ (in the case that $F_{\mathrm{a}} F_{\mathrm{g}}$ is assumed to be 1) values only in the DDI condition (Supplemental Figs. 4C and D). Results showed that the alteration of these parameters greatly affected the simulated concentration profile of CER.

Sensitivity Analyses of the Parameters of CER and GEM/GEMglu under DDI Conditions with GEM. To search for better described DDI-related parameters of CER with GEM from reported in vitro values, sensitivity analyses were carried out for $K_{\text {i_oATP1B1 }}$ of GEM/GEM-glu, $K_{\mathrm{i} \text {,app_CYP2C8 }}$, and $k_{\text {inact_CYP2C8 }}$ of GEM-glu under DDI conditions with GEM (Supplemental Figs. 5A-C). Upper and lower limits of the range of the in vitro parameters in Supplemental Figs. 5A-C were set by maximum and minimum value of the respective in vitro parameters. As shown in Supplemental Fig. 5A, the simulated CER concentration profile was changed by alteration of $K_{\mathrm{i} \_ \text {OATP1B } 1}$. On the other hand, CER concentration was not affected by changing the parameters related to the mechanism-based inhibition, $K_{\mathrm{i}, \text { app_CYP2C8 }}$ and $k_{\text {inact_CYP2C8, of }}$ GEM-glu (Supplemental Figs. 5B and C). To examine a relationship between the blood concentrations of GEM/GEM-glu and the in vitro $K_{\mathrm{i}}$ value, unbound blood concentration-time profiles of GEM (black line) and GEM-glu (blue line) were simulated (Supplemental Fig. 5E) by using their parameters described in Table 2 and Supplemental Table 3. As shown in this graph, the unbound blood concentration of GEM-glu

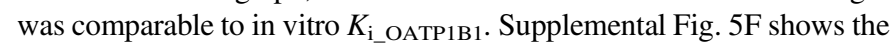
fraction of active CYP2C8 simulated using the previously reported geometric mean of in vitro values $\left(K_{\mathrm{i} \text {,app_CYP2C8 }}\right.$ and $k_{\text {inact_CYP2C8 }}$ of GEM-glu, and $k_{\text {deg_CYP2C8}}$ ) (Supplemental Tables 3 and 5), indicating that the fraction of active CYP2C8 after multiple administration of GEM was quite low, less than 0.05 .

The $f_{\mathrm{m} \_ \text {CYP2C8 }}$ value of CER was previously estimated to be 0.61 in in vitro studies using human liver microsomes (Shitara et al., 2003, 2004). In contrast, it was already reported that the activities of metabolism by human liver microsomes greatly depended on the buffer components and their concentration and thus the in vitro $f_{\mathrm{m}}$ value is suggested to be greatly altered by buffer condition (Kudo et al., 2016). We therefore conducted a sensitivity analysis for $f_{\mathrm{m} \_\mathrm{CYP} 2 \mathrm{C} 8}$ (Supplemental Fig. 5D). An increase in $t_{1 / 2}$ was observed with an increase in the value of $f_{\mathrm{m} \_\mathrm{CYP} 2 \mathrm{C} 8}$, and the simulated CER curves came closer to the clinical data with an increase in $f_{\mathrm{m} \_\mathrm{CYP} 2 \mathrm{C} 8}$ value.
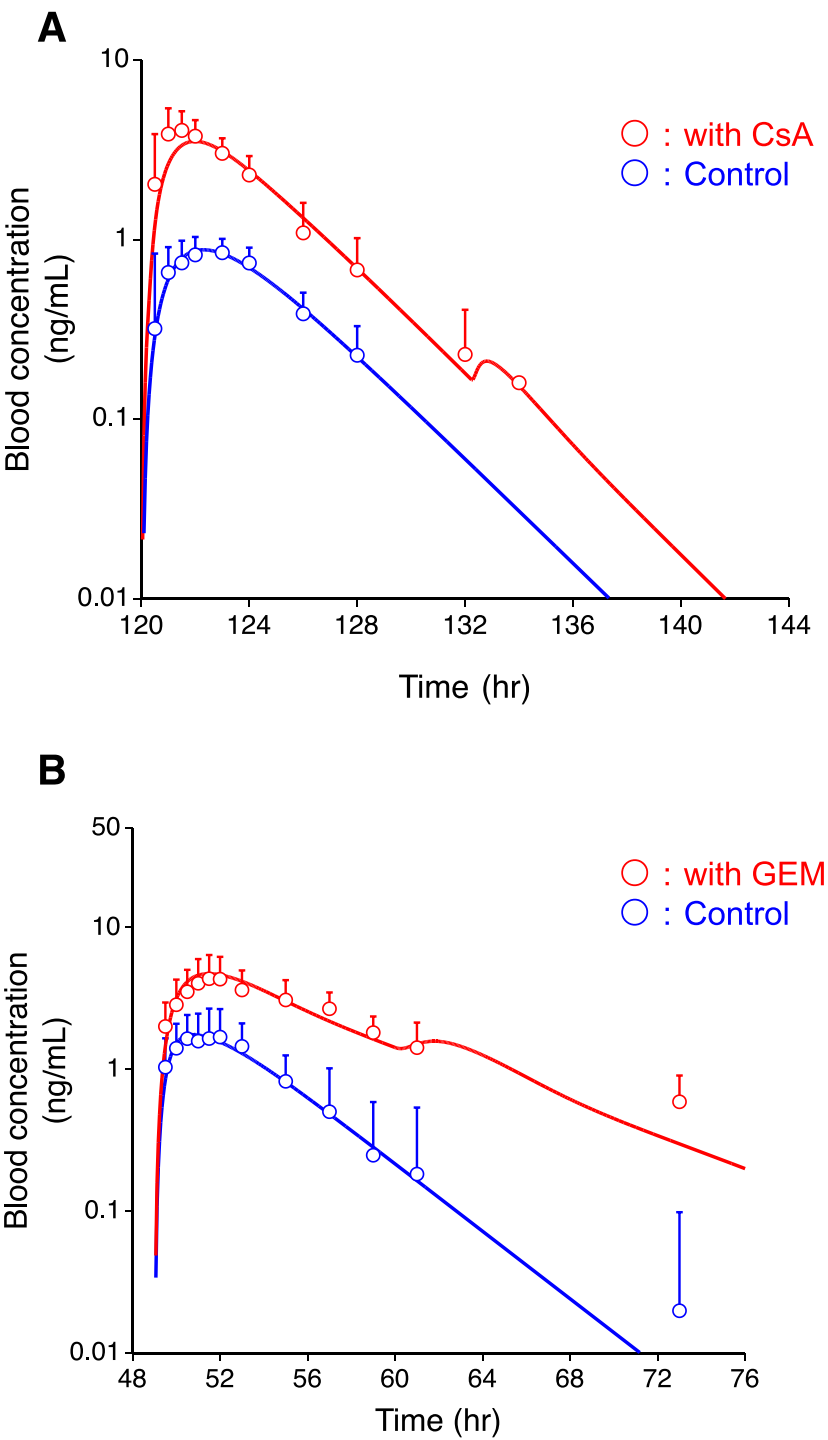

Fig. 4. Simulated blood concentration-time profile of CER under control and DDI conditions using the best described in vitro parameter sets (Table 3) after the sensitivity analyses (Supplemental Figs. 4 and 5) of model parameters to explain PK data under DDI conditions. (A) Control and DDI with CsA after single oral administration of $0.2 \mathrm{mg}$ CER (111 mg of CsA was orally administered twice daily for 6 days). The solid blue and red lines represent the simulated time course under control and DDI conditions, respectively. The open circles represent the observed values (Mück et al., 1999). (B) Control and DDI with GEM after single oral administration of $0.3 \mathrm{mg}$ CER (600 mg of GEM was orally administered twice daily for 3 days). The solid blue and red lines represent the simulated time course under control and DDI conditions, respectively. The open circles represent the observed values (Backman et al., 2002). 
Simulation of the Blood Concentration-Time Profile of CER under Control and DDI Conditions Using the Best Described In Vitro Parameter Sets after the Sensitivity Analyses. Finally, the CER concentration profiles under control and DDI conditions with CsA (Fig. 4A) or GEM (Fig. 4B) were simulated using the best described in vitro parameter sets (Table 3 ) after the sensitivity analyses together with the parameters estimated in the present study described in Tables 1 and 2 and Supplemental Tables 2 and 4. As observed in these graphs, the obtained concentration profiles of CER under control and DDI conditions with CsA or GEM agreed well with the clinically observed data (Mück et al., 1999; Backman et al., 2002). In this study, sensitivity analyses for DDI between CER and GEM were performed using the most optimized parameter set of GEM/GEM-glu only, but blood concentration-time profiles of CER under control and DDI conditions with GEM were simulated using the best described in vitro parameter sets after sensitivity analyses (Table 3 ) with the second to fifth mostoptimized parameter sets (Supplemental Fig. 6).

\section{Discussion}

CER pharmacokinetics were reported to be altered by DDI with CsA or GEM (Mück et al., 1999; Backman et al., 2002). Coadministration of CsA increased the AUC but not the elimination half-life $\left(t_{1 / 2}\right)$ of CER, whereas GEM increased both AUC and $t_{1 / 2}$. In this study, we attempted to describe the transporter-/enzyme-mediated such complex DDIs of CER based on unified PBPK models as well as to investigate whether the DDIs can be quantitatively analyzed by a bottom-up approach.

After separate optimization, the PBPK models for CER and inhibitors were linked, taking into consideration the appropriate inhibitions of OATP1B1 and P450s. As shown in Supplemental Fig. 3, using the geometric means of in vitro values, simulated CER concentration profiles under DDI conditions were lower than the observed data. In contrast, after sensitivity analyses, the reproduced concentration-time profiles of CER obtained using the best described drug-dependent parameter sets (Table 3 ) under DDI conditions agreed well with the clinically observed data as shown in Fig. 4. These results indicate that it is quite possible to describe quantitatively the clinical DDIs based on a bottom-up approach, taking into consideration the plausible ranges of inhibition potency (e.g., from minimum to maximum of in vitro values).

In this analysis, the best described $K_{\mathrm{i} \_ \text {OATP1B } 1 \text {, with a minimum }}$ in vitro value of $0.014 \mu \mathrm{M}$, is nearly equal to the in vivo $K_{\mathrm{i}}$ for PTV $(0.0118 \mu \mathrm{M})$ and $F L V(0.00958 \mu \mathrm{M})$, suggesting the validity of the value (Yoshikado et al., 2016). Furthermore, the CER blood concentration profile under DDI was substantially changed by taking account of the intestinal inhibition of Pgp and CYP3A4, and the simulated CER curves came to fit better with an alteration of these values, suggesting that CsA has a high inhibitory potential against Pgp-mediated efflux and/or CYP3A4-mediated metabolism of CER in the intestine (Supplemental Figs. 4C and D). Tachibana et al. (2009) reported that a drug interaction number (DIN, defined as the ratio of inhibitor dose to inhibition constant) is useful in classifying the risk of intestinal DDI involving Pgp and CYP3A4. In that report, Pgp inhibitors with a DIN for Pgp greater than 27.9 1 and CYP3A4 inhibitors with a DIN for CYP3A4 greater than 9.41 were reported to have a high risk. For this CsA case, calculated DIN_Pgp and DIN_CYP3A4 were $54.291(111 \mathrm{mg} / 1.7 \mu \mathrm{M})$ (Yoshida et al., 2013)) and 24.88 (111 mg/3.71 $\mu \mathrm{M}$ (Kim et al., 2017)), indicating that the intestinal Pgp and CYP3A4 were strongly inhibited by CsA. Although further study using a more mechanistic model with consideration given to intestinal inhibition, such as a segregated flow model (Chow and Pang, 2013), ACAT model (Agoram et al., 2001) or ADAM model (Jamei et al., 2009a,b), is needed, these results suggest that the clinical DDI between CER and CsA was caused by inhibition of both hepatic OATP1B1 and intestinal Pgp and/or CYP3A4 by CsA.

According to Supplemental Fig. 5E, the simulated unbound blood concentration of GEM-glu was comparable to the range of in vitro $K_{\mathrm{i} \_ \text {OATP1B1(glu) }}$. In addition, the best described $K_{\mathrm{i} \_ \text {OATP1B1(GEM) }}$ and $K_{\mathrm{i} \_ \text {OATP1B1(glu) }}$ were minimum in vitro values of 4.00 and $1.48 \mu \mathrm{M}$, respectively. These values closely match the in vivo $K_{\mathrm{i}}$ for repaglinide (14.2 and $5.48 \mu \mathrm{M}$ ), suggesting the validity of these values (Kudo et al., 2013). The fraction of active CYP2C8 after multiple administration of GEM was less than 0.05. Furthermore, the simulated CER curves came to fit better with an increase in $f_{\mathrm{m}_{-} \mathrm{CYP} 2 \mathrm{C} 8}$. (Supplemental Fig. 5D). These results suggest that the in vivo DDI between CER and GEM was due mainly to inhibition of CYP2C8-mediated metabolism by GEM-glu and partly to OATP1B1-mediated uptake by GEM/GEM-glu. Moreover, they also suggest the importance of properly estimating the contributions of each P450 to total metabolism to quantitatively describe the DDI.

Our group already reported that five tanks in series model (five-liver model) was useful for the hepatic elimination of high-clearance drugs (Watanabe et al., 2009); however, it was unclear whether the five-liver model is also robust for the drugs with relatively high $F_{\mathrm{h}}$, such as CER (Mück et al., 1997). Therefore, by using a one- or three-liver model, the concentration-time profile of CER was refitted to the clinically observed data. Then, DDI simulations between CER and CsA in one- and threeliver models were performed in the same way as the five-liver model (Supplemental Fig. 3) to examine the impact of the number of liver compartments (Supplemental Fig. 7). Optimized parameters are shown in Supplemental Tables 6-8. As shown in the figure, there is little

TABLE 3

In vitro parameter sets after sensitivity analyses

\begin{tabular}{|c|c|c|c|}
\hline Parameters & Units & Values & Sources \\
\hline $\begin{array}{l}\text { DDI conditions with CsA } \\
K_{\mathrm{i}} \text {. }\end{array}$ & $\mu \mathrm{M}$ & 0.014 & Supplemental Fig. 4A \\
\hline $\begin{array}{l}K_{\mathrm{i}} \text { OATP1B1 of CsA } \\
K_{\mathrm{i}} \text { CYP3A4 of CsA }\end{array}$ & & 3.71 & Supplemental Fig. 4B \\
\hline & $\mu \mathrm{M}$ & 0791 (control)/1 (DDI) & Supplemental Fig. 4B \\
\hline $\begin{array}{l}\mathrm{F}_{\mathrm{a}} \mathrm{F}_{\mathrm{g}} \text { of CER } \\
k_{\mathrm{a}} \text { of } \mathrm{CER}\end{array}$ & & $\begin{array}{c}0.791 \text { (control)/1 (DDI) } \\
0.789 \text { (control)/25 (DDI) }\end{array}$ & Supplemental Fig. 4C \\
\hline $\begin{array}{l}k_{\mathrm{a}} \text { of CER } \\
\mathrm{fm} \text { CYP2C } 8 \text { of CER }\end{array}$ & $\mathrm{h}$ & 0.789 (control)/2.5 (DDI) & Supplemental Fig. 4D \\
\hline $\begin{array}{l}\text { fm_CYP2C8 of CER } \\
\text { DDI conditions with GEM }\end{array}$ & & 0.85 & Supplemental Fig. 5D \\
\hline Ki_OATP1B1 of GEM & $\mu \mathrm{M}$ & 4.00 & Supplemental Fig. 5A \\
\hline$K_{\mathrm{i} \_\mathrm{OATP} 1 \mathrm{~B} 1(\mathrm{glu})}^{-} / \mathrm{K}_{\mathrm{i} \_ \text {OATP1B } 1(\mathrm{GEM})}$ & & 0.371 & $\begin{array}{l}\text { Ratio of geometric mean of in } \\
\quad \text { vitro } K_{\mathrm{i}} \text { (Supplemental Table 5) }\end{array}$ \\
\hline$K_{\mathrm{i} \_ \text {OATP1B1 }}$ of GEM-glu & $\mu \mathrm{M}$ & 1.48 & \\
\hline$K_{\mathrm{i}, \mathrm{app} \_\mathrm{CYP} 2 \mathrm{C} 8}$ of GEM-glu & $\mu \mathrm{M}$ & 26.7 & Supplemental Fig. 5B \\
\hline$k_{\text {inact_CYP2C8 }}$ of GEM-glu & $/ \mathrm{h}$ & 4.05 & Supplemental Fig. 5C \\
\hline$f_{\mathrm{m} \_\mathrm{CYP} 2 \mathrm{C} 8}$ of $\mathrm{CER}$ & & 0.85 & Supplemental Fig. 5D \\
\hline
\end{tabular}

CsA, cyclosporine A; DDI, drug-drug interactions; GEM, gemfibrozil. 
difference in reproducibility among all liver models, indicating the robustness of the five-liver model.

Although the existence of the biliary excretion and/or metabolism of CER in the mouse, rat, and dog was reported previously (Boberg et al., 1998), the typical second peak was not reported in the clinical data (Mück et al., 1997, 1999; Backman et al., 2002). Thus, fitting analyses to the control data (intravenous/oral) and subsequently the DDI simulation with CsA were performed using the model without EHC in the same way as with EHC to examine whether EHC is needed (Supplemental Fig. 8). Optimized parameters are shown in Supplemental Tables 9 and 10. In these analyses, the same scaling factor for $K_{\mathrm{p}}$ values of muscle, skin, and adipose were set and optimized to agree with the distribution phase of intravenous data. As shown in Supplemental Fig. 8, fitted concentrationtime profiles of the intravenous/oral data (Supplemental Fig. 8A) and control data in the DDI study (Supplemental Figs. 8B-E; blue lines) also agreed well with the clinically observed data. On the other hand, the simulated concentration-time profile under DDI conditions obtained using the best described parameter sets in the non-EHC model (Supplemental Fig. 8F) was lower than the simulated concentration-time profile obtained in the EHC model (Fig. 4A) and the observed data even after sensitivity analyses for $K_{\mathrm{iOATP} 1 \mathrm{~B} 1}$ and $K_{\mathrm{iCYP} 3 \mathrm{~A} 4}$ of CsA (Supplemental Figs. 8B and C) and $F_{\mathrm{a}} F_{\mathrm{g}}$ and/or $k_{\mathrm{a}}$ of CER (Supplemental Figs. 8D and $\mathrm{E}$ ). These results indicate that the clinically observed data were not perfectly reproduced using the model without EHC, suggesting that EHC of cerivastatin in humans exists to some extent. Although further study is needed to validate the adequate number of EHC compartments in cerivastatin cases, we selected the three EHCs model in these analyses for the reason that three compartments were thought to be enough to describe the delay in transit between the hepatocyte and intestine and for consistency with our previous study.

In conclusion, the results of this study revealed that the degree of complex DDIs between CER and the inhibitors CsA and GEM, involving both transporters and metabolic enzymes, could be quantitatively described based on a bottom-up approach and some sensitivity analyses, although variabilities of reported in vitro $K_{\mathrm{i}}$ values should be always kept in mind. The PBPK models and approaches in this study would be applicable to the prediction of other DDIs involving both transporters and metabolic enzymes.

\section{Acknowledgments}

We thank the members of the PKPD seminar and the Analysis \& Pharmacokinetics Research Laboratories, Drug Discovery Research, Astellas Parma Inc. (Dr. Kenji Tabata, Dr. Yasuhisa Nagasaka, and Dr. Tsuyoshi Minematsu).

\section{Authorship Contributions}

Participated in research design: Yao, Toshimoto, Kim, Yoshikado, Sugiyama. Performed data analysis: Yao, Toshimoto, Kim.

Wrote or contributed to the writing of the manuscript: Yao, Toshimoto, Kim, Yoshikado, Sugiyama.

\section{References}

Agoram B, Woltosz WS, and Bolger MB (2001) Predicting the impact of physiological and biochemical processes on oral drug bioavailability. Adv Drug Deliv Rev 50(Suppl 1):S41-S67. Aoki Y, Hayami K, Sterck HD, and Konagaya A (2014) Cluster Newton method for sampling multiple solutions of underdetermined inverse problems: application to a parameter identification problem in pharmacokinetics. SIAM J Sci Comput 36:B14-B44.

Backman JT, Kyrklund C, Neuvonen M, and Neuvonen PJ (2002) Gemfibrozil greatly increases plasma concentrations of cerivastatin. Clin Pharmacol Ther 72:685-691.

Boberg M, Angerbauer R, Kanhai WK, Karl W, Kern A, Radtke M, and Steinke W (1998) Biotransformation of cerivastatin in mice, rats, and dogs in vivo. Drug Metab Dispos 26: 640-652.

Brewer HB, Jr (2003) Benefit-risk assessment of Rosuvastatin 10 to 40 milligrams. Am J Cardiol 92 (4B):23K-29K.

Chow EC and Pang KS (2013) Why we need proper PBPK models to examine intestine and liver oral drug absorption. Curr Drug Metab 14:57-79.

Davies B and Morris T (1993) Physiological parameters in laboratory animals and humans. Pharm Res 10:1093-1095.
Deguchi T, Watanabe N, Kurihara A, Igeta K, Ikenaga H, Fusegawa K, Suzuki N, Murata S, Hirouchi M, Furuta Y, et al. (2011) Human pharmacokinetic prediction of UDPglucuronosyltransferase substrates with an animal scale-up approach. Drug Metab Dispos 39: $820-829$.

Furberg CD and Pitt B (2001) Withdrawal of cerivastatin from the world market. Curr Control Trials Cardiovasc Med 2:205-207.

Hisaka A and Sugiyama Y (1998) Analysis of nonlinear and nonsteady state hepatic extraction with the dispersion model using the finite difference method. J Pharmacokinet Biopharm 26: 495-519.

Honkalammi J, Niemi M, Neuvonen PJ, and Backman JT (2011) Mechanism-based inactivation of CYP2C8 by gemfibrozil occurs rapidly in humans. Clin Pharmacol Ther 89:579-586.

Izumi S, Nozaki Y, Komori T, Maeda K, Takenaka O, Kusano K, Yoshimura T, Kusuhara H, and Sugiyama Y (2013) Substrate-dependent inhibition of organic anion transporting polypeptide 1B1: comparative analysis with prototypical probe substrates estradiol-17 $\beta$-glucuronide, estrone-3-sulfate, and sulfobromophthalein. Drug Metab Dispos 41:1859-1866.

Izumi S, Nozaki Y, Maeda K, Komori T, Takenaka O, Kusuhara H, and Sugiyama Y (2015) Investigation of the impact of substrate selection on in vitro organic anion transporting polypeptide 1B1 inhibition profiles for the prediction of drug-drug interactions. Drug Metab Dispos 43:235-247.

Jamei M, Marciniak S, Feng K, Barnett A, Tucker G, and Rostami-Hodjegan A (2009a) The Simcyp population-based ADME simulator. Expert Opin Drug Metab Toxicol 5: 211-223.

Jamei M, Turner D, Yang J, Neuhoff S, Polak S, Rostami-Hodjegan A, and Tucker G (2009b) Population-based mechanistic prediction of oral drug absorption. AAPS J 11:225-237.

Kim SJ, Toshimoto K, Yao Y, Yoshikado T, and Sugiyama Y (2017) Quantitative analysis of complex drug-drug interactions between repaglinide and cyclosporin A/gemfibrozil using physiologically based pharmacokinetic models with in vitro transporter/enzyme inhibition data. J Pharm Sci 106:2715-2726.

Kimoto E, Li R, Scialis RJ, Lai Y, and Varma MV (2015) Hepatic disposition of gemfibrozil and its major metabolite gemfibrozil 1-O- $\beta$-glucuronide. Mol Pharm 12:3943-3952.

Kivistö KT, Zukunft J, Hofmann U, Niemi M, Rekersbrink S, Schneider S, Luippold G, Schwab M, Eichelbaum M, and Fromm MF (2004) Characterisation of cerivastatin as a P-glycoprotein substrate: studies in P-glycoprotein-expressing cell monolayers and mdrla/b knock-out mice. Naunyn Schmiedeberos Arch Pharmacol 370:124-130.

Kudo T, Hisaka A, Sugiyama Y, and Ito K (2013) Analysis of the repaglinide concentration increase produced by gemfibrozil and itraconazole based on the inhibition of the hepatic uptake transporter and metabolic enzymes. Drug Metab Dispos 41:362-371.

Kudo T, Ozaki Y, Kusano T, Hotta E, Oya Y, Komatsu S, Goda H, and Ito K (2016) Effect of buffer conditions on CYP2C8-mediated paclitaxel $6 \alpha$-hydroxylation and CYP3A4-mediated triazolam $\alpha$ - and 4-hydroxylation by human liver microsomes. Xenobiotica 46:241-246.

Li R, Barton HA, and Varma MV (2014) Prediction of pharmacokinetics and drug-drug interactions when hepatic transporters are involved. Clin Pharmacokinet 53:659-678.

Mück W (2000) Clinical pharmacokinetics of cerivastatin. Clin Pharmacokinet 39:99-116.

Mück W, Mai I, Fritsche L, Ochmann K, Rohde G, Unger S, Johne A, Bauer S, Budde K, Roots I, et al. (1999) Increase in cerivastatin systemic exposure after single and multiple dosing in cyclosporine-treated kidney transplant recipients. Clin Pharmacol Ther 65:251-261.

Mück W, Ritter W, Ochmann K, Unger S, Ahr G, Wingender W, and Kuhlmann J (1997) Absolute and relative bioavailability of the HMG-CoA reductase inhibitor cerivastatin. Int $J$ Clin Pharmacol Ther 35:255-260.

Ogilvie BW, Zhang D, Li W, Rodrigues AD, Gipson AE, Holsapple J, Toren P, and Parkinson A (2006) Glucuronidation converts gemfibrozil to a potent, metabolism-dependent inhibitor of CYP2C8: implications for drug-drug interactions. Drug Metab Dispos 34:191-197.

Rodgers T and Rowland M (2006) Physiologically based pharmacokinetic modelling 2: predicting the tissue distribution of acids, very weak bases, neutrals and zwitterions. J Pharm Sci 95: $1238-1257$.

Shitara Y, Hirano M, Sato H, and Sugiyama Y (2004) Gemfibrozil and its glucuronide inhibit the organic anion transporting polypeptide 2 (OATP2/OATP1B1:SLC21A6)-mediated hepatic uptake and CYP2C8-mediated metabolism of cerivastatin: analysis of the mechanism of the clinically relevant drug-drug interaction between cerivastatin and gemfibrozil. $J$ Pharmacol Exp Ther 311:228-236.

Shitara Y, Itoh T, Sato H, Li AP, and Sugiyama Y (2003) Inhibition of transporter-mediated hepatic uptake as a mechanism for drug-drug interaction between cerivastatin and cyclosporin A. J Pharmacol Exp Ther 304:610-616.

Shitara Y and Sugiyama Y (2006) Pharmacokinetic and pharmacodynamic alterations of 3-hydroxy-3-methylglutaryl coenzyme A (HMG-CoA) reductase inhibitors: drug-drug interactions and interindividual differences in transporter and metabolic enzyme functions. Pharmacol Ther 112:71-105.

Tachibana T, Kato M, Watanabe T, Mitsui T, and Sugiyama Y (2009) Method for predicting the risk of drug-drug interactions involving inhibition of intestinal CYP3A4 and P-glycoprotein. Xenobiotica 39:430-443.

Toshimoto K, Tomaru A, Hosokawa M, and Sugiyama Y (2017) Virtual clinical studies to examine the probability distribution of the AUC at target tissues using physiologically-based pharmacokinetic modeling: application to analyses of the effect of genetic polymorphism of enzymes and transporters on irinotecan induced side effects. Pharm Res 34:1584-1600.

Varma MV, Lin J, Bi YA, Kimoto E, and Rodrigues AD (2015) Quantitative rationalization of gemfibrozil drug interactions: consideration of transporters-enzyme interplay and the role of circulating metabolite gemfibrozil 1-O-ß-glucuronide. Drug Metab Dispos 43: $1108-1118$.

Watanabe T, Kusuhara H, Maeda K, Shitara Y, and Sugiyama Y (2009) Physiologically based pharmacokinetic modeling to predict transporter-mediated clearance and distribution of pravastatin in humans. J Pharmacol Exp Ther 328:652-662.

Watanabe T, Kusuhara H, Watanabe T, Debori Y, Maeda K, Kondo T, Nakayama H, Horita S, Ogilvie BW, Parkinson A, et al. (2011) Prediction of the overall renal tubular secretion and hepatic clearance of anionic drugs and a renal drug-drug interaction involving organic anion transporter 3 in humans by in vitro uptake experiments. Drug Metab Dispos 39:1031-1038. Wooltorton E (2001) Bayer pulls cerivastatin (Baycol) from market. CMAJ 165:632.

Yang J, Liao M, Shou M, Jamei M, Yeo KR, Tucker GT, and Rostami-Hodjegan A (2008) Cytochrome p450 turnover: regulation of synthesis and degradation, methods for determining rates, and implications for the prediction of drug interactions. Curr Drug Metab 9:384-394. 
Yoshida K, Maeda K, Kusuhara H, and Konagaya A (2013) Estimation of feasible solution space using Cluster Newton Method: application to pharmacokinetic analysis of irinotecan with physiologically-based pharmacokinetic models. BMC Syst Biol 7 (Suppl 3):S3

Yoshida K, Maeda K, and Sugiyama Y (2012) Transporter-mediated drug-drug interactions involving OATP substrates: predictions based on in vitro inhibition studies. Clin Pharmacol Ther 91:1053-1064.

Yoshikado T, Toshimoto K, Nakada T, Ikejiri K, Kusuhara H, Maeda K, and Sugiyama Y (2017) Comparison of methods for estimating unbound intracellular-to-medium concentration ratios in rat and human hepatocytes using statins. Drug Metab Dispos 45 779-789.
Yoshikado T, Yoshida K, Kotani N, Nakada T, Asaumi R, Toshimoto K, Maeda K, Kusuhara H, and Sugiyama Y (2016) Quantitative analyses of hepatic OATP-mediated interactions between statins and inhibitors using PBPK modeling with a parameter optimization method. Clin Pharmacol Ther 100:513-523.

Address correspondence to: Dr. Yuichi Sugiyama, Sugiyama Laboratory, RIKEN Innovation Center, 1-7-22 Suehiro-cho, Tsurumi-ku, Yokohama, Kanagawa 230 0045, Japan. E-mail: ychi.sugiyama@riken.jp 